\title{
Quantification of R-Fuzzy Sets
}

\author{
Arjab Singh Khuman ${ }^{\mathrm{a}, *}$, Yingjie Yang ${ }^{\mathrm{a}}$, Robert John ${ }^{\mathrm{b}}$ \\ ${ }^{a}$ Centre for Computational Intelligence, De Montfort University, The Gateway, Leicester LE1 9BH, UK \\ ${ }^{b}$ Automated Scheduling, Optimisation and Planning (ASAP), Nottingham University, Nottingham NG8 $1 B B$, UK
}

\begin{abstract}
The main aim of this paper is to connect R-Fuzzy sets and type-2 fuzzy sets, so as to provide a practical means to express complex uncertainty without the associated difficulty of a type-2 fuzzy set. The paper puts forward a significance measure, to provide a means for understanding the importance of the membership values contained within an R-fuzzy set. The pairing of an R-fuzzy set and the significance measure allows for an intermediary approach to that of a type-2 fuzzy set. By inspecting the returned significance degree of a particular membership value, one is able to ascertain its true significance in relation, relative to other encapsulated membership values. An R-fuzzy set coupled with the proposed significance measure allows for a type-2 fuzzy equivalence, an intermediary, all the while retaining the underlying sentiment of individual and general perspectives, and with the adage of a significantly reduced computational burden. Several human based perception examples are presented, wherein the significance degree is implemented, from which a higher level of detail can be garnered. The results demonstrate that the proposed research method combines the high capacity in uncertainty representation of type-2 fuzzy sets, together with the simplicity and objectiveness of type-1 fuzzy sets. This in turn provides a practical means for problem domains where a type-2 fuzzy set is preferred but difficult to construct due to the subjective type-2 fuzzy membership.

Keywords: R-Fuzzy Sets, Rough Sets, Fuzzy Membership, Significance, Type-2 Equivalence
\end{abstract}

2010 MSC: 00-01, 99-00

\section{Introduction}

The work undertaken by Yang and Hinde (2010) first proposed the notion of R-fuzzy sets, the capital 'R' distinguishes it from r-fuzzy, which was proposed by Li et al. (1996), yet another approach to encapsulate uncertainty. The membership value of an element of an R-fuzzy set is represented as a rough set. Rfuzzy sets are an extension of fuzzy set theory that allows for the uncertain fuzzy membership value to be encapsulated within the bounds of an upper and lower rough approximation. The lower bound contains

\footnotetext{
*Corresponding author. Tel: +44 (0) 1163486857

Email addresses: Arjab.Khuman@dmu.ac.uk (Arjab Singh Khuman), yyang@dmu.ac.uk (Yingjie Yang), Robert.John@nottingham.ac.uk (Robert John)
} 
the membership values agreed upon by all, whereas the upper bound contains membership values agreed upon by at least one. Many different variations of uncertainty exist in the real world, all of which have their own associated difficulties in exacting crisp, clear and concise information. The notion of sets in a classical sense incorporates the use of crisp boundaries, either a complete inclusion of an element or object, or complete exclusion, as was stated by Cantor (1895). A set was created with the specific role of being able to evaluate a member of either belonging, or not-belonging. As it was later understood, human nature and inferencing does not work in this way, human inferencing is not preformed in such a crisp and precise manner, everything is vague to some extent. With this realisation, the concept of a classical set did not seem 15 a fitting synthesis for human decision making. Something else was needed, ergo, the notion of mereology (Lesniewski, 1929), which considered the idea of an object being partially included in a set. In mathematics, crisp understandings are needed for precise reasoning, this becomes problematic when concepts based on natural language are considered. Abstract terms with inherent vagueness and ambiguity are often used in our daily communications, therefore reasoning cannot be based solely on classical logic. This gave rise to the concept of fuzzy theory (Zadeh, 1965, 1975, 1972). Fuzzy logic adopts the mantra of mereology, whereby an element can belong to a set to some degree, inclusive of its membership function; $\mu_{A}(x): \mathbb{U} \rightarrow[0,1]$. Here the element $x$ belongs to the set $A$ by a returned value equal to or within the range of $[0,1]$.

One problem that still exists is that of deriving a crisp membership function for a standard type-1 fuzzy set, as it may involve vagueness and ambiguity, hence why there have been many extensions developed in an attempt to overcome this pitfall (Deschrijver and Kerre, 2003). Atanassov intuitionistic fuzzy sets (Atanassov, 1986), where a degree of membership and degree of non-membership are presented. Shadowed sets (Pedrycz, 1998), where the evaluation of a membership is scored as either (1), (0) or belonging to the shadowed region $[0,1]$. Interval-valued fuzzy sets (Sambuc, 1975), where the membership of an individual element is characterised as an interval itself. Type-2 fuzzy sets (Mendel and John, 2002), where the membership function itself is a type-1 fuzzy set. These new approaches involve the use of intervals, multiple parameters and additional fuzzy sets to describe the uncertain membership function values of fuzzy sets. However, the shortcomings that these approaches share is that they do not recognise the difference between values within their intervals or shadow areas.

The ongoing interest in type-2 fuzzy logic as a higher order form of fuzzy logic, has received a lot of attention. The use of interval type-2 fuzzy logic and the generalised approach of type-2 fuzzy logic has garnered much interest, particularly for its ability to handle higher degrees of uncertainty. As a result, its application areas are varied, but considerable work has been undertaken in clustering, classification and pattern recognition. A thorough review of type-2 fuzzy logic applications was undertaken by Melin and Castillo (2014). The majority of the applications reviewed are based on interval-type 2 fuzzy logic, which has less associated computational overhead compared to the more computationally expensive generalised type-2 approach. As every value in the secondary grade of membership is given a membership of 1 , only the 
foot-print-of-uncertainty is often used. It can be agreed upon that a generalised approach will indeed cater and allow for better management of handling uncertainty, compared to that of the interval type-2 approach. However, the associated complexities are often the reason that an interval type-2 approach is ultimately chosen.

As the work proposed in this paper can be seen as a bridge to cater for a generalised type-2 approach, it is noteworthy to extend a mention to some of the other current works that allow for generalised type-2 equivalence. The work proposed by Melin et al. (2014), applied the theory of alpha planes which were used to help create generalised type-2 fuzzy logic for image detection. Wagner and Hagras (2010, 2013) propose the use of z-slices as means to reduce the computational burden. Mendel et al. (2009) proposed the use of alpha planes to represent type-2 fuzzy logic sets.

R-fuzzy sets tackles the problem from a different perspective, via the use of rough sets to approximate the uncertain fuzzy membership function values of a fuzzy set. By utilising the approximation that rough sets employs, R-fuzzy sets allows for the membership values of the entire populous to be included. Rough sets themselves allow for a different perspective to that of fuzzy sets with regards to uncertainty. A rough perspective is with relation to ambiguity, a lack of information, whereas a fuzzy approach is more akin to vagueness, a lack of sharp definable boundaries. As a result there have been several hybridisation between fuzzy sets and rough sets to allow for greater versatility in encapsulating uncertainty; (Bodjanova, 2007), (Deng et al., 2007), (Dubois, 1980), (Dubois and Prade, 1990), (Huynh and Nakamori, 2005), (Jensen and Shen, 2008, 2009), (Nanda and Majumdar, 1992), (Pawlak and Skowron, 2007), (Radzikowska and Kerre, 2002), (Sun et al., 2014), (Wu et al., 2003), (Xu et al., 2012), (Zeng et al., 2015), all of which mainly incorporate the use of equivalence and similarity relations. The notion of R-fuzzy was the first approach that used rough sets to approximate the membership functions of fuzzy sets (Yang and Hinde, 2010).

Section 2 will go on to provide the foundational preliminaries for fuzzy sets, rough sets and R-fuzzy sets, along with their associated notations. A worked example involving human perception regarding noise pollution using an R-fuzzy approach is demonstrated. Section 3 introduces the newly derived significance degree. The noise pollution example is further extended by the implementation of the significance measure, to quantify the meaning and intent of the encapsulated membership values. In addition, a human perception based example regarding visualisation is also put forward. Section 4 describes the relationships that exist between R-fuzzy sets, the significance measure and traditional fuzzy sets. The equivalence between the coupling of an R-fuzzy set and the significance measure, to that of a type-2 fuzzy set is remarked upon. Section 5 provides the reader with remarks, where the strengths and weaknesses of the proposed research are discussed, along with theoretical comparisons to other approaches. Section 6 draws out the conclusion and summarises upon the advantages of using an R-fuzzy approach in conjunction with a significance degree measure for human perception based modelling. Final remarks, possible enhancements for future work are also discussed. 


\subsection{Motivation}

The motivation for this paper comes from the desire to extend the applicability of R-fuzzy sets. As a result, the novelty of this paper is with regards to providing a means to quantify the importance of each validator for values contained within the lower approximation, as the returned value should be an absolute 1 . Equally, if the membership values were completely disregarded the returned value would be an absolute 0 , as they would not be included within the rough set. Any returned value within the interval $[0,1]$ signifies that the membership value has some importance to some degree. This echoes the sentiment of a typical type-1 fuzzy set and in doing so, enhances the overall existing robustness and versatility of R-fuzzy sets, increasing its scope for applicability. The better understood a problem, the more better equipped the solution.

According to Klir and Wierman (1998) there exist three kinds of general uncertainty. Real world problems often involve uncertainty, from an empirical level, uncertainty is often associated with any type of measurement. Resolution can be a cause for concern when involving exactness; 0.1 is different from 0.01 as it to 0.001 , and so on. From the cognitive level, uncertainty exists in the vagueness and ambiguity associated with natural language. Your understanding of a word may not have to be exact match to the person you are conversing with, suffice to say, an overlap of an understanding can still act as an agreement of the sentiment nonetheless. At the social level, uncertainty can be used to ones advantage, where it is often simulated by individuals for different purposes; privacy, secrecy and propriety (Klir and Wierman, 1998).

There could be several root causes for the existence of uncertainty. The information associated to the problem may be inherently noisy or incomplete, riddled with contradictions, vague and ambiguous. These deficiencies may result in sub-faceted aspects of uncertainty, uncertainty within uncertainty.

Therefore the categorised three states of uncertainty are given as vagueness, associated to fuzzy with respect to imprecise, vague boundaries of fuzzy sets. Imprecision, this is with regards to nonspecificty of the cardinalities of sets and their alternatives. Finally, discord, with regards to strife which expresses conflicts and contradictions of the various sets of alternatives (Klir and Wierman, 1998; Klir and Folger, 1988).

Klir and Wierman (1998) then go onto divide the aforementioned three main types of uncertainness into two distinct classes, fuzziness and ambiguity. These remarks are also echoed by Berenji (1988). The need for higher dimensionality for uncertainty encapsulation makes a generalised type-2 fuzzy logic approach very appealing. If one could lessen the burden of complexities of a type- 2 approach, it would allow for a greater scope of applicability. The works undertaken by Wagner and Hagras (2010, 2013) Melin et al. (2014) and Mendel et al. (2009) have all attempted to circumvent this problem. As it has been described, an R-fuzzy approach coupled with the significance measure allows for the distribution of the contained fuzzy membership values to be quantified. The continuous perspective mentioned earlier allows one to take the discrete understating of the problem and translate it to a continuous projection, via the use of customised membership functions as seen in Example 1 \& Example 2. The ability to distinguish a membership value 
from one another allows discernibility to be maintained. We are no longer concerned with loss of information, as would be the case if an interval approach was used. An R-fuzzy and significance measure approach allows for greater detailed information to be inferred from, which allows for better uncertainty management.

\section{Preliminaries}

The paper will now go on to present some of the core definitions needed in order to grasp the concepts being discussed.

\subsection{Fuzzy Set Theory Preliminaries}

We begin with fuzzy set theory, the most fundamental aspect of fuzzy set theory is its understanding of numbers. A fuzzy number is ideal for describing linguistic phenomena, where an exact description of its state is unknown. For example, the following statement, 'it's roughly 'pm' contains uncertainty, as allowances either side of 7 are included. This allows for a fuzzy number to be described using linguistic hedges (Zadeh, 1972), such as; nearly, almost, around and so on. Hedges act as operators, allowing for modification of the meaning and intent of the fuzzy set. It can be stated that any interpretation involving a fuzzy view is an extension of a fuzzy number. This is true for a crisp number, an interval number, a number which is reference to about a point, or an interval which describes how near it is to a point. A fuzzy number and a fuzzy set are very closely related, both mathematically and descriptively.

Definition 1 (Fuzzy set (Zadeh, 1965)): Let $\mathbb{U}$ represent the universe and let $A$ be a set in $\mathbb{U}(A \subseteq \mathbb{U})$. The fuzzy set $A$ is a set of ordered pairs given by the following expression:

$$
A=\left\{\left\langle x, \mu_{A}(x)\right\rangle \mid x \in \mathbb{U}\right\}
$$

Where $\mu_{A}: \mathbb{U} \rightarrow[0,1]$ is the membership function and the value returned by $\mu_{A}(x)$ quantifies the belongingness of element $x$ with respect to fuzzy set $A$. Replacing $\sum$ with $\int$ would provide for a continuous fuzzy set. In essence, the process of associating a membership value to an element is known as fuzzification. If $\mu_{A}(x)=1$, this signifies complete and absolute inclusion, whereas $\mu_{A}(x)=0$ signifies complete and absolute exclusion from the fuzzy set. Any real value in the range $[0,1]$ signifies that it belongs in part to the set by some degree.

Using multiple fuzzy sets and variations of membership functions, one is able to comprehensively encapsulate a given abstract concept. Such concepts may regard perception, in which case a universal assumption may not always be the case; what is beautiful to some people may not be beautiful to all. Quantifying the degree of inclusion of an object based on a modelled concept, allows one to gauge the perception of each object. The presented interpretation of fuzzy set theory is distinct from probability theory, however the literature is rife with works that describe the close relation between the two. Fuzzy is not probabilistic 
in nature, but more similar to possibilistic sets, fuzzy sets and possibilistic sets can indeed simulate probabilities as mentioned in Drakopoulos (1995). Fuzzy has been related to both probability and possibility in several works; (Coletti and Scozzafava, 2004), (Drakopoulos, 1995), (Pourahmadi-Nakhli et al., 2013), (Nguyen, 1997), (Purba, 2014), (Roisenberg et al., 2009), (Zadeh, 1999). As is the versatility of fuzzy, it has also been hybridised with different paradigms other than rough theory such as neural networks; (Balas et al., 2010), (Bosque et al., 2014), (Lin and Wai, 2002), (Otadi, 2014), (Pedrycz et al., 2001). Fuzzy has also been applied to genetic computing and genetic algorithms; (Mendes et al., 2012), (Nasab, 2014), (Nasir et al., 2014). There has also been a marrying together between fuzzy and grey systems; (Lian, 2012), (Rajmohan et al., 2013), (Salmeron and Gutierrez, 2012).

\subsection{Rough Set Theory Preliminaries}

Rough set theory associates itself with imperfect knowledge, tackling the problem of ambiguity and vagueness via the use of a boundary region. If the boundary region of an approximated set is empty, then one can conclude that the set being approximated is crisp. If the boundary region is non-empty, then one can assume that the set being approximated is rough and inexact (Pawlak, 1982). A non-empty boundary region means that our knowledge regarding the set being approximated is not sufficient to define the set precisely and exactly. In essence, the concept of a rough set deals with the synthesis of an approximation with regards to the classificatory analysis of a data set, by framing a given concept via the use of lower and upper approximations. Assuming that every object which belongs to the universe has some associated information regarding it, objects characterised by the same information make themselves indiscernible from one another. The indiscernibility relation provides the basis from which approximations can be made, as the relations themselves are relative to the attributes providing the information regarding the object. Objects which can be classified as belonging to the set being approximated with absolute certainty are placed in the lower approximation, whereas objects which cannot be classified with absolute certainty are placed into the upper approximation. The boundary region is the difference between the upper and lower approximation, the larger the cardinality of the boundary set, the greater the imprecision of the approximated set. Using the notion of approximations, one is able to frame a given concept and it is precisely this aspect of rough sets that has been factored into R-fuzzy sets; the approximation of fuzzy membership values.

Rough set theory can be further utilised for data exploration, reduction, reasoning and rule extraction. Propositional functions with relation to Bayes' theorem have been implemented into rough set theory as a means to understand and explain the rules generated (Pawlak, 1982, 1998), (Pawlak and Skowron, 2007). Thus providing truth values regarding strength, certainty and coverage. It is this aspect of quantification that provides the inspiration for the newly proposed significance measure. The qualities available from rough set theory can be applied to both standard information systems and decision systems. An information system is a pair; $\Lambda=(\mathbb{U}, A)$, where $\mathbb{U}$ is a non-empty finite set of objects referred to as the universe. $A$ is a non-empty 
finite set of attributes such that $a: \mathbb{U} \rightarrow V_{a}$ for every $a \in A$. The set $V_{a}$ is the value set for $a$. If the data set represents that of a decision system, then the notation would make use of the decision attribute; $\Lambda=(\mathbb{U}, A \cup\{d\})$, where $d \notin A$. A data set may contain a large amount of information, some of which may be superfluous. Rough sets employs the notion of equivalence and indiscernibility relations to reduce this overhead. Assume that we have a binary relation $R \subseteq X \times X$, where $R$ is referred to as the equivalence relation. The equivalence class of an element $x \in X$ contains all objects $y \in X$ such that $x R y$.

Definition 2 (Equivalence relation (Pawlak, 1998, 2002)): Assume that $\Lambda=(\mathbb{U}, A)$ is an information system and with any $B \subseteq A$ there is an associated equivalence relation $\operatorname{IND}_{\Lambda}(B)$, given by the formal expression:

$$
\operatorname{IND}_{\Lambda}(B)=\left\{\left(x, x^{\prime}\right) \in \mathbb{U}^{2} \mid \forall a \in X a(x)=a\left(x^{\prime}\right)\right\}
$$

Where $\operatorname{IND}_{\Lambda}(B)$ is referred to as the $B$-indiscernibility relation. If $\left(x, x^{\prime}\right) \in \operatorname{IND}_{\Lambda}(B)$, then it can be agreed that objects $x$ and $x^{\prime}$ are indiscernible from one another by the attributes from $B$. An equivalence relation allows one to partition the data set, which in turn can be used to build new subsets of the universe. One may then like to approximate a given concept based on its relation to an equivalence class. The notion of approximations allows one to further inspect a given concept.

Definition 3 (Approximations (Pawlak, 1998, 2002)): Assume that $\Lambda=(\mathbb{U}, A)$ is an information system and that $B \subseteq A$ and $X \subseteq \mathbb{U}$. One can approximate set $X$ with the information contained in $B$ via a lower and upper approximation set.

The lower approximation is the set of all objects that absolutely belong to set $X$ with respect to $B$. It is the union of all equivalence classes in $[x]_{B}$ which are contained within the target set $X$, and is given by the formal expression:

$$
\begin{gathered}
\underline{B} X=\left\{x \mid[x]_{B} \subseteq X\right\} \\
\underline{B}(x)=\bigcup_{x \in \mathbb{U}}\{B(x): B(x) \subseteq X\}
\end{gathered}
$$

The upper approximation is the set of all objects which can be classified as being possible members of set $X$ with respect to $B$. It is the union of all equivalence classes that have a non-empty intersection with the target set $X$, and is given by the formal expression:

$$
\begin{gathered}
\bar{B} X=\left\{x \mid[x]_{B} \cap X \neq \emptyset\right\} \\
\bar{B}(x)=\bigcup_{x \in \mathbb{U}}\{B(x): B(x) \cap X \neq \emptyset\}
\end{gathered}
$$

The boundary region is the set that contains all objects that cannot be decisively categorised as belonging to $X$ with respect to $B$. It is defined by the difference between the upper approximation Eq. (4) and the 
lower approximation Eq. (3), and is given by the formal expression:

$$
B N(X)=\bar{B}(X)-\underline{B}(X)
$$

Definition 4 (Rough sets (Yao, 1996)): Assume that the pair, apr $=(\mathbb{U}, B)$ is an approximation space on $\mathbb{U}$ and assume that $\mathbb{U} / B$ denotes the set of all equivalence classes over $B$. The family of all definable sets in approximation space apr is denoted by $\operatorname{def}(a p r)$. Given two subsets $\underline{A}, \bar{A} \in \operatorname{def}(\operatorname{apr})$ with $\underline{A} \subseteq \bar{A}$, the pair $(\underline{A}, \bar{A})$ is called a rough set.

- if $x \in \underline{A}$ then $x \in(\underline{A}, \bar{A})$

- if $x \in \mathbb{U}-\bar{A}$ then $x \notin A$

- if $x \in \bar{A}$ and $x \notin \underline{A}$ then $x$ has an unknown relation to $(\underline{A}, \bar{A})$

If the approximated set is crisp the boundary region of the set is empty, else if the approximated set is rough the boundary region of the set is non-empty.

\subsection{R-Fuzzy Set Preliminaries}

R-fuzzy sets is yet another proposal for encapsulating uncertainty, one which frames its fuzzy membership values via the approximations defined in Definition 3.

Definition 5 (R-fuzzy sets (Yang and Hinde, 2010)): Let the pair apr $=\left(J_{x}, B\right)$ be an approximation space on a set of values $J_{x}=\left\{v_{1}, v_{2}, \ldots, v_{n}\right\} \subseteq[0,1]$, and let $J_{x} / B$ denote the set of all equivalence classes of $B$. Let $\left(\underline{M}_{A}(x), \bar{M}_{A}(x)\right)$ be a rough set in apr. An R-fuzzy set $A$ is characterised by a rough set as its membership function $\left(\underline{M}_{A}(x), \bar{M}_{A}(x)\right)$, where $x \in \mathbb{U}$, given by the formal expression:

$$
A=\left\{\left\langle x,\left(\underline{M}_{A}(x), \bar{M}_{A}(x)\right)\right\rangle \mid \forall x \in \mathbb{U}, \underline{M}_{A}(x) \subseteq \bar{M}_{A}(x) \subseteq J_{x}\right\}
$$

Similar to type-2 fuzzy sets and interval-valued fuzzy, an R-fuzzy set describes its membership using a set itself, which are values that satisfy the membership descriptor; if a membership value has an affinity to the descriptor, then it is included within the R-fuzzy set. For each $x_{i} \in \mathbb{U}$, there is an associated membership description $d\left(x_{i}\right)$ which describes the belongingness of the element $x_{i}$ to the set $A \subseteq \mathbb{U}$. Then $C$ is the set of available evaluation criteria. Each value $v \in J_{x}$ is evaluated by $c_{j} \in C$ to determine if it is described by the membership description for $x_{i}$ with respect to $A$. The result of the evaluation is given by a simple YES or NO. Obviously, evaluations which return a YES are accepted while evaluations which return a NO are ignored.

$$
v \stackrel{\left(d\left(x_{i}\right), c_{j}\right)}{\longrightarrow} \text { YES } \quad \text { or } \quad v \stackrel{\left(d\left(x_{i}\right), c_{j}\right)}{\longrightarrow} \mathrm{NO}
$$


For each pair $\left(\left(x_{i}\right), c_{j}\right)$ where $x_{i} \in \mathbb{U}$ and $c_{j} \in C$, a set $M_{c j}\left(x_{i}\right) \subseteq J_{x}$ is created, given by the formal expression:

$$
M_{c j}\left(x_{i}\right)=\left\{v \mid v \in J_{x}, v \stackrel{\left(d\left(x_{i}\right), c_{j}\right)}{\longrightarrow} \mathrm{YES}\right\}
$$

The lower approximation of the rough set $M\left(x_{i}\right)$ for the membership function described by $d\left(x_{i}\right)$ is given by:

$$
\underline{M}\left(x_{i}\right)=\bigcap_{j} M_{c j}\left(x_{i}\right)
$$

The upper approximation of the rough set $M\left(x_{i}\right)$ for the membership function described by $d\left(x_{i}\right)$ is given by:

$$
\bar{M}\left(x_{i}\right)=\bigcup_{j} M_{c j}\left(x_{i}\right)
$$

Therefore the rough set approximating the membership $d\left(x_{i}\right)$ for $x_{i}$ is given as:

$$
M\left(x_{i}\right)=\left(\bigcap_{j} M_{c j}\left(x_{i}\right), \bigcup_{j} M_{c j}\left(x_{i}\right)\right)
$$
perception of the noise levels for each of the 10 flights. These values have been collected and are presented in Table 1. 
Table 1: Human perception of noise based on 10 flights

\begin{tabular}{c|cccccccccc}
\cline { 2 - 10 }$\#$ & $f_{1}$ & $f_{2}$ & $f_{3}$ & $f_{4}$ & $f_{5}$ & $f_{6}$ & $f_{7}$ & $f_{8}$ & $f_{9}$ & $f_{10}$ \\
\hline $\mathrm{dB}$ & 10 & 20 & 30 & 50 & 40 & 70 & 20 & 80 & 30 & 60 \\
$\mu\left(f_{i}\right)$ & 0.00 & 0.14 & 0.29 & 0.57 & 0.43 & 0.86 & 0.14 & 1.00 & 0.29 & 0.71 \\
\hline \hline$p_{1}$ & $N N$ & $N N$ & $N N$ & $A C$ & $A C$ & $B N$ & $N N$ & $V N$ & $N N$ & $B N$ \\
$p_{2}$ & $N N$ & $N N$ & $A C$ & $A C$ & $A C$ & $B N$ & $N N$ & $V N$ & $A C$ & $B N$ \\
$p_{3}$ & $N N$ & $A C$ & $A C$ & $B N$ & $A C$ & $V N$ & $A C$ & $V N$ & $A C$ & $B N$ \\
$p_{4}$ & $N N$ & $N N$ & $N N$ & $A C$ & $A C$ & $B N$ & $N N$ & $V N$ & $N N$ & $B N$ \\
$p_{5}$ & $N N$ & $A C$ & $A C$ & $A C$ & $A C$ & $B N$ & $A C$ & $V N$ & $A C$ & $B N$ \\
$p_{6}$ & $N N$ & $N N$ & $A C$ & $A C$ & $A C$ & $V N$ & $N N$ & $V N$ & $A C$ & $B N$ \\
\hline
\end{tabular}

The abbreviated terms contained within the table can be understood as meaning:

$$
N N \rightarrow \text { Not Noisy } \quad A C \rightarrow \text { Acceptable } \quad B N \rightarrow \text { A Bit Noisy } \quad V N \rightarrow \text { Very Noisy }
$$

To construct a fuzzy set, one takes the values contained in $N$, and then inserts them into a simple linear function given as follows:

$$
\mu\left(f_{i}\right)=\frac{l_{i}-l_{\min }}{l_{\max }-l_{\min }}
$$

Where $l_{i}$ is reference to the noise level of flight $f_{i} \cdot l_{\max }$ and $l_{\min }$ provide the normalising scope and are the maximum and minimum values contained in $N$. After completion, one is presented with a fuzzy set containing precise fuzzy membership values for each of the 10 flights:

$$
\begin{array}{lllll}
\mu\left(f_{1}\right)=0.00 & \mu\left(f_{2}\right)=0.14 & \mu\left(f_{3}\right)=0.29 & \mu\left(f_{4}\right)=0.57 & \mu\left(f_{5}\right)=0.43 \\
\mu\left(f_{6}\right)=0.86 & \mu\left(f_{7}\right)=0.14 & \mu\left(f_{8}\right)=1.00 & \mu\left(f_{9}\right)=0.29 & \mu\left(f_{10}\right)=0.71
\end{array}
$$

It is not always possible to know the exact noise level of a particular flight, nor do people need to know the exact levels in their communication (Yang and Hinde, 2010). An R-fuzzy approach provides an answer to the question, how to express a fuzzy membership function if the exact noise level is unknown or not given? If for example, we know that flight $f_{11}$ has an associated abstract description of being Acceptable (AC), how can this be encapsulated using fuzzy membership values? A standard fuzzy type-1 approach would assign it a precise value, but this does not fully appreciate the differences in perception individuals may have for a given flight, as can be seen from Table 1. A particular flight can be perceived in a multitude of ways; what is acceptable to some may not be acceptable to all. Rather than neglecting to include a particular response as it may go against the grain of common held interpretations, it would be more ideal to encapsulate all perceptions; here is where R-fuzzy comes into its own. 
Now that we have established our membership values based on the recorded noise levels and the linear function given in Eq. (11), we have our membership set:

$$
J_{x}=\{0.00,0.14,0.29,0.57,0.43,0.86,0.14,1.00,0.29,0.71\}
$$

If we know that $f_{11}$ is Acceptable, we can set the descriptor to $d\left(f_{11}\right)=$ 'Acceptable', where its membership has to satisfy this description. The evaluation criterion is decided by each individual from set $C$. Each value $v \in J_{x}$ is evaluated against $p_{j} \in C$ to conclude if it fits with the description given for $d\left(f_{11}\right)$ and for $f_{11} \in \mathbb{U}$, using:

$$
v \stackrel{\left(d\left(x_{i}\right), c_{j}\right)}{\longrightarrow} \text { YES }
$$

For each $p_{i} \in C$ there is a corresponding row in Table 1. For the columns where there is a match with the descriptor given for $d\left(f_{11}\right)$, its corresponding flights will provide the membership values in accordance to their noise levels. Using the description of Acceptable for flight $f_{11}$ and the values provided by the individuals in set $C$, we can construct a subset of values, $M_{p j}\left(f_{11}\right) \subseteq J_{x}$. For example, inspecting $p_{1}$, one can see that flights $f_{4}$ and $f_{5}$ are the only flights that satisfy the descriptor $d\left(f_{11}\right)=$ 'Acceptable'. As a result, we take the corresponding membership values from $J_{x}$ for $f_{4}$ and $f_{5}$, which gives a subset of values, $M_{p 1}\left(f_{11}\right)=\{0.57,0.43\}$. For $p_{2}$, the descriptor is satisfied by flights $f_{3}, f_{4}, f_{5}$ and $f_{9}$. This results in subset $M_{p 2}\left(f_{11}\right)=\{0.29,0.57,0.43\}$, where instances of duplication are ignored. This process is repeated for all objects of set $C$, the results of which are given as follows:

$$
\begin{array}{lll}
M_{p 1}\left(f_{11}\right)=\{0.57,0.43\} & M_{p 2}\left(f_{11}\right)=\{0.29,0.57,0.43\} & M_{p 3}\left(f_{11}\right)=\{0.14,0.29,0.43\} \\
M_{p 4}\left(f_{11}\right)=\{0.57,0.43\} & M_{p 5}\left(f_{11}\right)=\{0.14,0.29,0.57,0.43\} & M_{p 6}\left(f_{11}\right)=\{0.29,0.57,0.43\}
\end{array}
$$

Once we have collected our subsets $M_{p j}\left(f_{11}\right)$, we can now apply the notion of approximations. Starting with the lower approximation and using Eq. (8), we inspect each subset to find any membership value that occurs in each and every subset. As a result $\{0.43\}$ is the only membership value that satisfies this requirement, therefore it is the only value to be contained in the lower approximation of the rough set. If no such membership value existed, whereby it was not included in all generated subsets, then resulting rough set would contain an empty lower approximation. The upper approximation Eq. (9), contains values that have been considered to be valid with relation to the descriptor. This essentially means that all instances contained within the subsets are placed into the upper approximation, where the duplications are removed $\{0.14,0.29,0.43,0.57\}$. One will notice that the lower approximation value of $\{0.43\}$ is also contained, this is understandable as Eq. (6) clearly states that $\underline{M}_{A}(x) \subseteq \bar{M}_{A}(x)$. Any value contained in the lower approximation will also be contained within the upper approximation. The actual rough set approximating the uncertain membership for $d\left(f_{11}\right)$ is constructed using Eq. (10), therefore we are presented with:

$$
M\left(f_{11}\right)=(\{0.43\},\{0.14,0.29,0.43,0.57\})
$$


This result alludes to the fact that the membership value 0.43 is agreed upon by all and that its corresponding flights are Acceptable, as it is the only value to be contained within the lower approximation. Also, the flights associated with $0.14,0.29$ and 0.57 are also considered as Acceptable by some but not all.

\section{Quantification}

This section describes the newly proposed significance measure derived specifically for R-fuzzy sets. Example 1 is further enhanced via the addition of the significance measure, as it provides a means for understanding the intent of the contained membership values.

\subsection{Significance}

The significance measure proposed in this paper is based on and takes its inspiration from the certainty factor employed by traditional rough set theory, which itself is based on a variation of Bayes' theorem (Pawlak, 1998). Rough set theory allows one to employ to use of strength, certainty and coverage factors, each one providing an insight into a particular rule or fact. As they are associated with decision systems and rule induction, they cannot be translated over to R-fuzzy sets without modification. With every decision rule $A \rightarrow_{x} D$ there is an associated certainty factor of the decision rule, where $A$ is the rule, $D$ is the decision, and supp is a frequency count. The certainty factor presented from a rough set perspective is given as follows:

$$
\operatorname{cer}_{x}(A, D)=\frac{|A(x) \cap D(x)|}{|A(x)|}
$$

It can be viewed as a conditional probability that $y$ belongs to $D(x)$ given that $y$ belongs to $A(x)$, symbolically $\pi_{x}(D \mid A)$. If $\operatorname{cer}_{x}(A, D)=1$, then $A \rightarrow_{x} D$ will be called a certain decision rule, if $0<$ $\operatorname{cer}_{x}(A, D)<1$ the decision rule will be referred to as an uncertain decision rule. To allow for the use of a certainty factor in an R-fuzzy context, one has to remove the notion of rules, induction of rules or quantification of rules. Therefore making the new significance measure relative to the subset of all values based on $M_{p j}(x) \subseteq J_{x}$.

Definition 6 (Degree of significance): Using the same notations presented in Definition 5 that described an R-fuzzy set, assume that an R-fuzzy set $M\left(x_{i}\right)$ has already been created, and that a membership set of values $J_{x}$ and a criteria set $C$ are known. Assume that the total number of subsets generated for a given R-fuzzy set is given by $|N|$, and that $S_{v}$ is the number of subsets that contain the specified membership value being inspected. As each value $v \in J_{x}$ is evaluated by $c_{j} \in C$, the significance measure therefore counts the number of instances that $v$ occurred over $|N|$, given by the formal expression:

$$
\gamma_{\bar{A}}\{v\}=\frac{S_{v}}{|N|}
$$


The significance measure expresses the conditional probability that $v \in J_{x}$ belongs to the R-fuzzy set $M\left(x_{i}\right)$, given by its descriptor $d\left(x_{i}\right)$. The value returned will be presented as a standard fraction, where the denominator $|N|$ represents the cardinality of the total number of generated subsets. The numerator $S_{v}$ is indicative of the number of instances that the inspected membership value $v \in J_{x}$ occurred. As $|N|$ provides the scope of the domain in terms of magnitude, $S_{v}$ will never exceed $|N|$. In essence providing a normalised output which in turn can be translated into any real value, where $\gamma_{\bar{A}}\{v\}: J_{x} \rightarrow[0,1]$ is the membership function, much like that of Definition 1. If the value returned by $\gamma_{\bar{A}}\{v\}=1$, then that particular membership value has been agreed upon by all in the criteria set $C$. As a result one will know that it absolutely belongs to the lower approximation; as for it to be included, the entire populous must agree:

$$
\underline{M}_{A}=\left\{\gamma_{\bar{A}}\{v\}=1 \mid v \in J_{x} \subseteq[0,1]\right\}
$$

Much like before how a lower approximation is a subset of the upper approximation, any membership value with a significance degree of $\gamma_{\bar{A}}\{v\}=1$, will also be included within the upper approximation. If $\gamma_{\bar{A}}\{v\}=0$, it can be concluded that absolutely no-one perceived that particular membership value to satisfy the descriptor. If $0<\gamma_{\bar{A}}\{v\}<1$, then that particular membership value has some significance to some degree relative to the descriptor $d\left(x_{i}\right)$. As a result this particular value will knowingly be contained within the upper approximation:

$$
\bar{M}_{A}=\left\{\gamma_{\bar{A}}\{v\}>0 \mid v \in J_{x} \subseteq[0,1]\right\}
$$

These interpretations echo the sentiments of fuzzy set theory as presented in Definition 1, whereby an element can be described by its membership function such that it returns any real number in the range $[0,1]$. Except instead of representing the belongingness of an object to a particular set, the significance degree returns the measure of significance, with relation to its descriptor $d\left(x_{i}\right)$, based on its conditional probability of distribution. Eq. (13) can be rewritten so that the collected significance degrees constitute a set, given by the following expression:

$$
\bar{A}=\left\{\left\langle v, \gamma_{\bar{A}}\{v\}\right\rangle \mid v \in J_{x} \subseteq[0,1]\right\}
$$

Where $\bar{A}$ is set describing the distribution of a specified descriptor $d(x)$, for which the generated R-fuzzy set was created for. It must be understood that fuzzy sets are not concerned with probability, merely the degree of belongingness (Drakopoulos, 1995). As the newly derived significance measure itself is based on Eq. (12), then the significance is associated with the conditional probability of distribution. The significance measure will in affect validate any value contained in the lower approximation as $\gamma_{\bar{A}}\{v\}=1$; as this is considered as an absolute truth agreed upon by all. $\gamma_{\bar{A}}\{v\}=0$ will validate that the inspected membership value was not considered significant to any degree by anyone. The greater the returned value for $\gamma_{\bar{A}}\{v\}$ the greater its significance with regards to the descriptor that the R-fuzzy set is being modelled for. Understanding the significance of any inspected membership value, can be used to understand the perception of the 
populous that it was generated from. R-fuzzy sets allow for every conceivable perception to be incorporated, that includes all possible outliers. The associated degrees of significance will quantify just how important or unimportant a membership value truly is based on the perceptions collected.

Referring back to Example 1, it would be meaningful if one was able to obtain the importance of the membership values contained within the upper approximation. If it can be established that a membership value was almost contained within the lower approximation, then one would be able to make allowances to cater for such an instance. By using Eq. (13), we are able to provide a means of quantification which in turn provides a significance coefficient equal to or within the range of $[0,1]$. The higher the value the higher its significance, and the more individuals that agree with its sentiment. The newly derived significance measure is relatively simple, in that it is a statistical method for counting the significance of a particular membership in relation to $J_{x}$ and its descriptor. For each membership value contained in $J_{x}$, we simply count the number of occurrences each instance occurred for each of the subsets created for $M_{p j}\left(f_{11}\right)$. As a result, we can obtain the following significance coefficient values for each of the membership values contained in $J_{x}$, given as follows:

$$
\begin{aligned}
\gamma \overline{A C}\{0.00\} & =\frac{0}{6}=0.00 \\
\gamma \overline{A C}\{0.29\} & =\frac{4}{6}=\frac{2}{3}=0.67 \\
\gamma \overline{A C}\{0.57\} & =\frac{5}{6}=0.83 \\
\gamma \overline{A C}\{0.86\} & =\frac{0}{6}=0.00
\end{aligned}
$$

$$
\begin{aligned}
\gamma{ }_{\overline{A C}}\{0.14\} & =\frac{2}{6}=\frac{1}{3}=0.33 \\
\gamma \overline{A C}\{0.43\} & =\frac{6}{6}=1.00 \\
\gamma{ }_{\overline{A C}}\{0.71\} & =\frac{0}{6}=0.00 \\
\gamma_{\overline{A C}}\{1.00\} & =\frac{0}{6}=0.00
\end{aligned}
$$

A possible visualisation for when the descriptor is set to Acceptable, based on its returned degrees of significance for the values contained in the membership set $J_{x}$, is presented in Fig. 1.

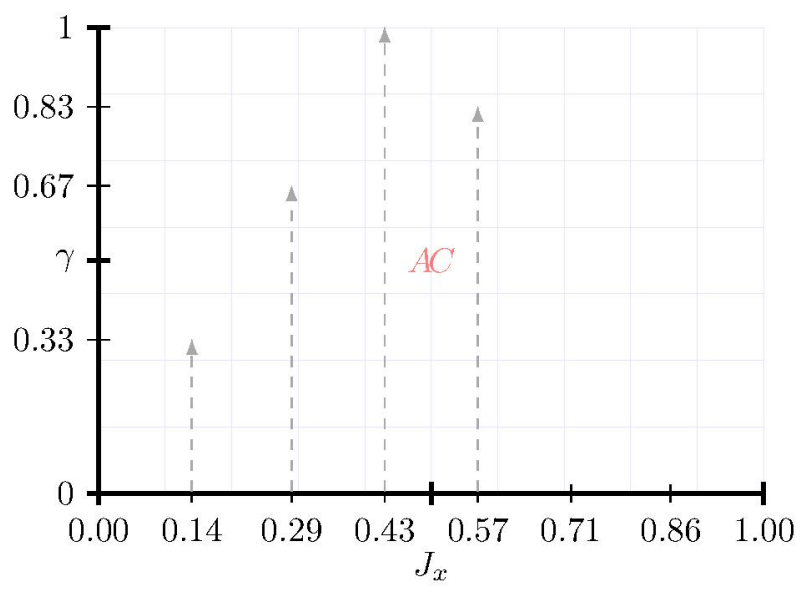

Figure 1: A discrete visualisation for Acceptable, based on the returned degrees of significance

The membership value 0.43 returns a degree of significance of $\gamma \overline{A C}\{0.43\}=1$, echoing the fact that it 
was agreed upon by all. Fig. 1 does indeed indicate and also validates that the membership value 0.43 was included within the lower approximation. For any value to score a significance of 1 satisfies the requirement of membership to the set representing the descriptor, if and only if the values for the significance degree provide the values for the membership function parameters. Fig. 1 presents a discretised interpretation of the degree of significance for the descriptor Acceptable, whereas Fig. 2 provides a continuous interpretation for the descriptor Acceptable.

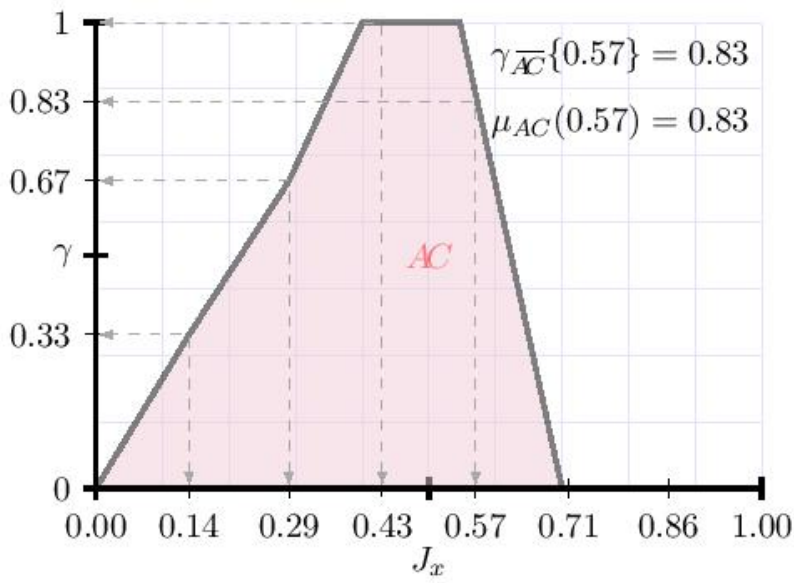

Figure 2: A possible continuous visualisation for Acceptable, based on the returned degrees of significance

One can easily infer from this continuous representation that the significance degrees are also the degrees of membership, akin to a fuzzy perspective. The parameters for the set were based on the returned degrees of significance, hence why the lines intersect through the apex of the stick heights for the generated degrees of significance. However, notice how Fig. 2 utilises a trapezoidal membership rather than the expected triangular membership. This was done to show that variation is still allowed and the choice for a trapezoidal 
value 0.42 not scoring a degree of 1 for its significance, as would be the case for 0.44 and so forth. It would seem more likely and indeed more plausible that a membership value of 0.42 and 0.44 would indeed be agree upon by all, especially if the triggered 0.43 was, as a result a trapezoidal membership was chosen. The apex of which is now an interval given by the range [0.43,0.55], a completely arbitrary assumption, one which to visualise a possible interpretation of how human perception given a descriptor, propagates through the membership values.

Inspecting the significance of each of the membership values, one is able to apply linguistic assumptions to describe them, such as:

$\gamma \overline{A C}\{0.00\}=0.00 \rightarrow$ Agreed upon by none

$\gamma \overline{A C}\{0.29\}=0.67 \rightarrow$ Agreed upon by the vast majority

$\gamma \overline{A C}\{0.57\}=0.83 \rightarrow$ Agreed upon by the vast majority

$\gamma \overline{A C}\{0.86\}=0.00 \rightarrow$ Agreed upon by none $\gamma \overline{A C}\{0.14\}=0.33 \rightarrow$ Agreed upon by a few

$\gamma \overline{A C}\{0.43\}=1.00 \rightarrow$ Agreed upon by all

$\gamma \overline{A C}\{0.71\}=0.00 \rightarrow$ Agreed upon by none

$\gamma \overline{A C}\{1.00\}=0.00 \rightarrow$ Agreed upon by none

Using simple statements, one is able to easily ascertain the intent of any membership value by referring to its description. The statements chosen were trivial, but further consideration could be included. Such as, the incorporation of a fuzzy set to indicate how strongly a membership value agrees to each of the statements. This would further enhance the ability to understand the significance of any membership value inspected.

If one was to use a traditional type-1 fuzzy approach to define the membership Acceptable, the average may be taken to represent it, in which case the returned value would be:

$$
\mu\left(f_{11}\right)=\frac{1}{17} \sum_{x \in \mathbb{U}} \mu(x) / x=0.40
$$

Which is the summation of each membership value contained in all the generated $M_{p j}\left(f_{11}\right) \in J_{x}$. Inspecting the value 0.40 , it is slightly less than the accepted and more reasonable 0.43 , and considerably less than the high scoring 0.57 , which was almost included in the lower approximation. Even if one was to apply interval-valued fuzzy sets, where the scope would be the most pessimistic lower bound and optimistic upper bound $[0.14,0.57]$, it is not possible to tell which values were agreed upon unanimously. This same problem is shared with Atanassov intuitionistic fuzzy sets. This harps back to the initial problem as described in 
Section I, that there is no distinction between the values in the interval regions or shadow areas, the value

itself loses its individuality. With regards to type-2 fuzzy sets, the example is too small so that a reliable membership distribution can be setup. If the shadowed set was to be used, the set Acceptable would be placed in the unknown region. One can see that in this instance, an R-fuzzy set is an ideal concept to use, maintaining uniqueness for all viable membership values.

If the descriptor for Example 1 was changed to $d\left(f_{11}\right)=$ 'Not Noisy', the following subsets $M_{p j}\left(f_{11}\right) \subseteq J_{x}$ would be generated:

$$
\begin{array}{ll}
M_{p 1}\left(f_{11}\right)=\{0.00,0.14,0.29\} & M_{p 2}\left(f_{11}\right)=\{0.00,0.14\} \\
M_{p 3}\left(f_{11}\right)=\{0.00\} & M_{p 4}\left(f_{11}\right)=\{0.00,0.14,0.29\} \\
M_{p 5}\left(f_{11}\right)=\{0.00\} & M_{p 6}\left(f_{11}\right)=\{0.00,0.14\}
\end{array}
$$

This would create the following R-fuzzy set:

$$
M\left(f_{11}\right)=(\{0.00\},\{0.00,0.14,0.29\})
$$

The associated degrees of significance using Eq. (13), would return the following results:

$$
\begin{array}{ll}
\gamma \frac{6}{N N}\{0.00\}=\frac{6}{6}=1.00 & \gamma \frac{\overline{N N}}{2}\{0.14\}=\frac{4}{6}=\frac{2}{3}=0.67 \\
\gamma \frac{2}{N N}\{0.29\}=\frac{2}{6}=\frac{1}{3}=0.33 & \gamma \frac{\overline{N N}}{3}\{0.43\}=\frac{0}{6}=0.00 \\
\gamma \frac{0}{N N}\{0.57\}=\frac{0}{6}=0.00 & \gamma \frac{0}{N N}\{0.71\}=\frac{0}{6}=0.00 \\
\gamma \frac{0}{N N}\{0.86\}=\frac{0}{6}=0.00 & \gamma \frac{}{N N}\{1.00\}=\frac{0}{6}=0.00
\end{array}
$$

A possible visualisation for when the descriptor is set to Not Noisy, based on its returned degrees of significance for the values contained in the membership set $J_{x}$, is presented in Fig. 3.

If the descriptor for Example 1 was changed to $d\left(f_{11}\right)=$ ' $A$ Bit Noisy', the following subsets $M_{p j}\left(f_{11}\right) \subseteq$ $J_{x}$ would be generated:

$$
\begin{array}{ll}
M_{p 1}\left(f_{11}\right)=\{0.86,0.71\} & M_{p 2}\left(f_{11}\right)=\{0.86,0.71\} \\
M_{p 3}\left(f_{11}\right)=\{0.57,0.71\} & M_{p 4}\left(f_{11}\right)=\{0.86,0.71\} \\
M_{p 5}\left(f_{11}\right)=\{0.86,0.71\} & M_{p 6}\left(f_{11}\right)=\{0.71\}
\end{array}
$$

This would create the following R-fuzzy set:

$$
M\left(f_{11}\right)=(\{0.71\},\{0.57,0.71,0.86\})
$$




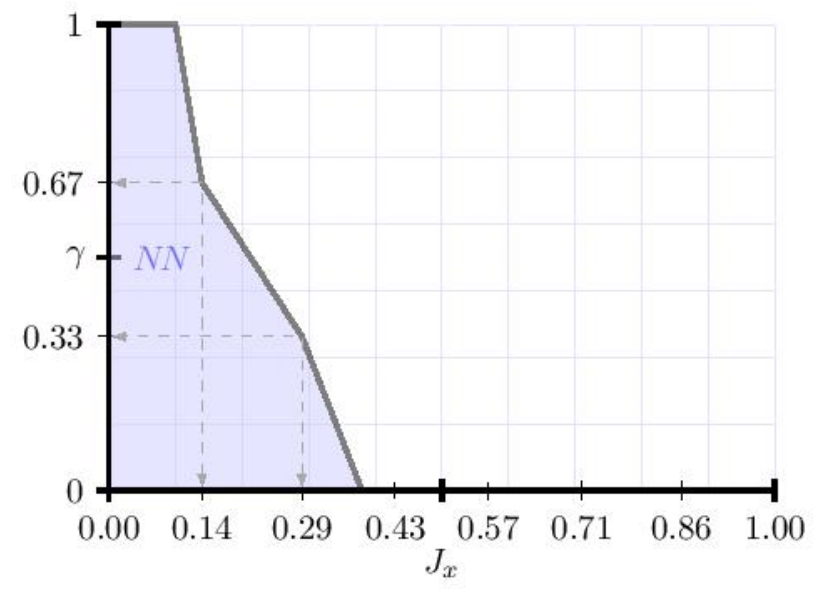

Figure 3: A possible continuous visualisation for Not Noisy, based on the returned degrees of significance

The associated significant coefficients using Eq. (13), would return the following results:

$$
\begin{aligned}
\gamma \overline{B N}\{0.00\} & =\frac{0}{6}=0.00 & \gamma \overline{B N}\{0.14\} & =\frac{0}{6}=0.00 \\
\gamma \overline{B N}\{0.29\} & =\frac{0}{6}=0.00 & \gamma \overline{B N}\{0.43\} & =\frac{0}{6}=0.00 \\
\gamma \overline{B N}\{0.57\} & =\frac{1}{6}=0.17 & \gamma \overline{B N}\{0.71\} & =\frac{6}{6}=1.00 \\
\gamma \overline{B N}\{0.86\} & =\frac{4}{6}=\frac{2}{3}=0.67 & \gamma \overline{B N}\{1.00\} & =\frac{0}{6}=0.00
\end{aligned}
$$

A possible visualisation for when the descriptor is set to A Bit Noisy, based on its returned degrees of significance for the values contained in the membership set $J_{x}$, is presented in Fig. 4.

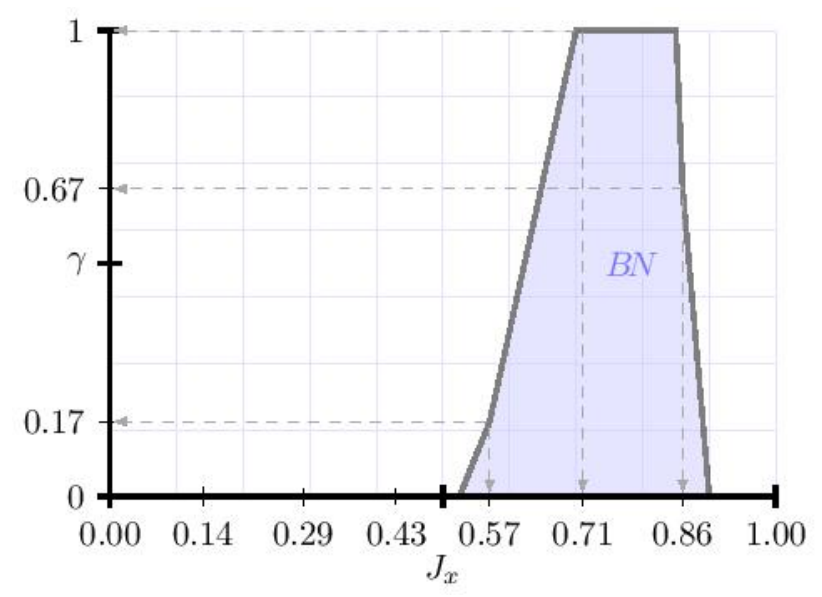

Figure 4: A possible continuous visualisation for A Bit Noisy, based on the returned degrees of significance

If the descriptor for Example 1 was changed to $d\left(f_{11}\right)=$ 'Very Noisy', the following subsets $M_{p j}\left(f_{11}\right) \subseteq J_{x}$ 
would be generated:

$$
\begin{array}{ll}
M_{p 1}\left(f_{11}\right)=\{1.00\} & M_{p 2}\left(f_{11}\right)=\{1.00\} \\
M_{p 3}\left(f_{11}\right)=\{0.86,1.00\} & M_{p 4}\left(f_{11}\right)=\{1.00\} \\
M_{p 5}\left(f_{11}\right)=\{1.00\} & M_{p 6}\left(f_{11}\right)=\{0.86,1.00\}
\end{array}
$$

This would create the following R-fuzzy set:

$$
M\left(f_{11}\right)=(\{1.00\},\{0.86,1.00\})
$$

The associated significant coefficients using Eq. (13), would return the following results:

$$
\begin{aligned}
\gamma_{\overline{V N}}\{0.00\} & =\frac{0}{6}=0.00 & \gamma_{\overline{V N}}\{0.14\} & =\frac{0}{6}=0.00 \\
\gamma_{\overline{V N}}\{0.29\} & =\frac{0}{6}=0.00 & \gamma_{\overline{V N}}\{0.43\} & =\frac{0}{6}=0.00 \\
\gamma_{\overline{V N}}\{0.57\} & =\frac{0}{6}=0.00 & \gamma_{\overline{V N}}\{0.71\} & =\frac{0}{6}=0.00 \\
\gamma_{\overline{V N}}\{0.86\} & =\frac{2}{6}=\frac{1}{3}=0.33 & \gamma_{\overline{V N}}\{1.00\} & =\frac{6}{6}=1.00
\end{aligned}
$$

A possible visualisation for when the descriptor is set to Very Noisy, based on its returned degrees of significance for the values contained in the membership set $J_{x}$, is presented in Fig. 5 .

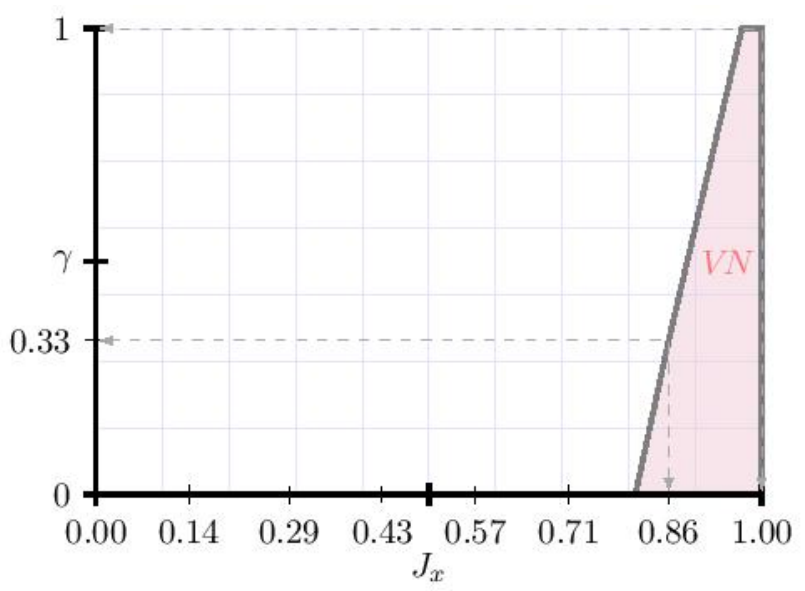

Figure 5: A possible continuous visualisation for Very Noisy, based on the returned degrees of significance

The example presented in this paper provides an introduction and an understanding into R-fuzzy sets. The addition of utilising the significance measure to inspect individual membership values allows one to better appreciate and conserve the diversity of perceptions being collected. The values contained within Table 2 are the collective significance measures for each of the specified descriptors. By placing the membership values contained in $J_{x}$ in ascending order, and so to the descriptors, starting with $N N$ through to 
Table 2: The collected significance degree and membership values for Example 1

\begin{tabular}{|c|c|c|c|c|c|c|c|c|}
\hline \multirow[b]{2}{*}{$J_{x}$} & \multicolumn{2}{|c|}{$N N$} & \multicolumn{2}{|c|}{$A C$} & \multicolumn{2}{|c|}{$B N$} & \multicolumn{2}{|c|}{$V N$} \\
\hline & $\gamma$ & $\mu$ & $\gamma$ & $\mu$ & $\gamma$ & $\mu$ & $\gamma$ & $\mu$ \\
\hline 0 & 1 & 1 & 0 & 0 & 0 & 0 & 0 & 0 \\
\hline 0.14 & 0.67 & 0.67 & 0.33 & 0.33 & 0 & 0 & 0 & 0 \\
\hline 0.29 & 0.33 & 0.33 & 0.67 & 0.67 & 0 & 0 & 0 & 0 \\
\hline 0.43 & 0 & 0 & 1 & 1 & 0 & 0 & 0 & 0 \\
\hline 0.57 & 0 & 0 & 0.83 & 0.83 & 0.17 & 0.17 & 0 & 0 \\
\hline 0.71 & 0 & 0 & 0 & 0 & 1 & 1 & 0 & 0 \\
\hline 0.86 & 0 & 0 & 0 & 0 & 0.67 & 0.67 & 0.33 & 0.33 \\
\hline 1 & 0 & 0 & 0 & 0 & 0 & 0 & 1 & 1 \\
\hline
\end{tabular}
perspectives are different as fuzzy is not associated with probability, whereas the significance degree returns the conditional distribution of probability.

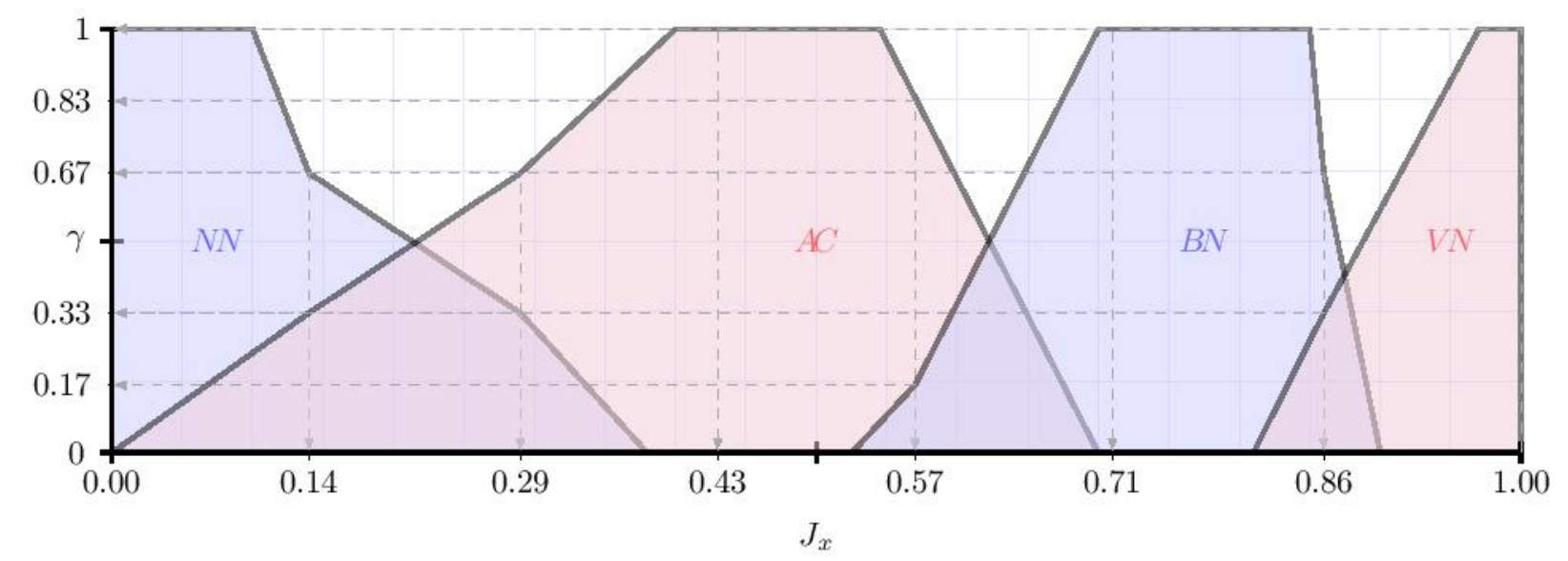

Figure 6: A continuous visualisation for Example 1, based on all the generated significance measures for the R-fuzzy sets of Not Noisy, Acceptable, A Bit Noisy \& Very Noisy

The plot presented in Fig. 6 shows the collective visualisation of sets based on all the generated R-fuzzy 
sets for Example 1. The $x$-axis, as before, represents the scope of the membership values $J_{x}$, and the $y$-axis represents the degree of significance. This provides a means to understand the entire collective perceptions of all involved for all descriptors for the entire domain. As the plot shows, each descriptor or perspective, does not follow uniformed symmetry. The sets themselves are not equal to one another, especially with regards to area and distribution. This synthesis of human perception is more probable and in keeping, than strict symmetrical uniformed sets. Paying particular interest to the set representing the descriptor Acceptable, one can see that the membership value 0.14 has a degree of significance of $\gamma \overline{A C}\{0.14\}=0.33$. The same membership value has a significance of $\gamma \overline{N N}\{0.14\}=0.67$ to the set describing the descriptor Not Noisy. The fact that the sets themselves are constructed via the stick heights of the triggered degrees of significance, allows one to establish an equivalence between a fuzzy set and the generated significance set. Albeit, an equivalence in the returned value and not the perspective the values are investigated from.

Example 2: Assume that $F=\left\{f_{1}, f_{2}, \ldots, f_{9}\right\}$ is a set containing 9 different colour swatches based on the color red:

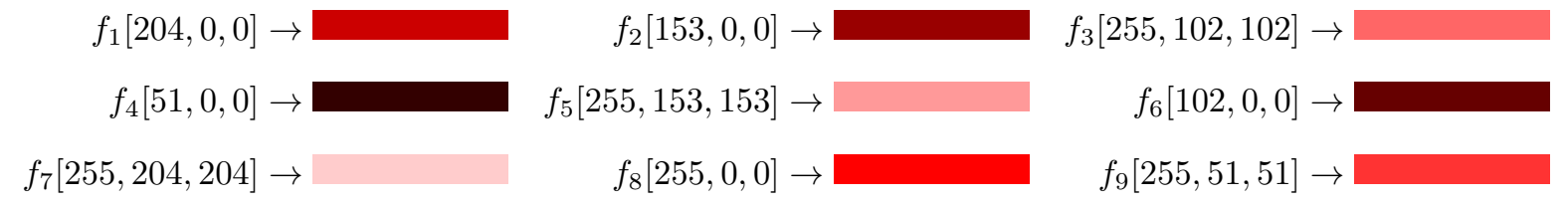

The RGB values are recorded from which the average is taken, the values are presented in $N=$ $\{68,51,153,17,187,34,221,85,119\}$. For sake of continuity the values are passed to Eq. (11) to provide the membership set:

$$
J_{x}=\{0.25,0.17,0.67,0.00,0.83,0.08,1.00,0.33,0.50\}
$$

Set $C=\left\{p_{1}, p_{2}, \ldots, p_{6}\right\}$ represents 6 individuals all of whom gave their perceived perceptions of the different variations for the colour red, these values have been collected and are presented in Table 3 . The abbreviated terms contained within the table can be understood as thusly:

$$
L R \rightarrow \text { Light Red } \quad R \rightarrow \text { Red } \quad D R \rightarrow \text { Dark Red }
$$

Assume that the $10^{\text {th }}$ swatch presented to the test group was described as being Red, and that the RGB values were not known. The question of which membership values could one use to describe such a descriptor, can be easily answered. The resulting R-fuzzy set for when the descriptor is set to Red would be:

$$
M\left(f_{11}\right)=(\{0.33\},\{0.17,0.25,0.33,0.5,0.67\})
$$

Using the degree of significance as given by Eq. (13), one is able to ascertain the significance for all 
Table 3: Human based perception for the colour red

\begin{tabular}{c|ccccccccc}
\cline { 2 - 9 } & $f_{1}$ & $f_{2}$ & $f_{3}$ & $f_{4}$ & $f_{5}$ & $f_{6}$ & $f_{7}$ & $f_{8}$ & $f_{9}$ \\
\hline \hline$p_{1}$ & $D R$ & $D R$ & $L R$ & $D R$ & $L R$ & $D R$ & $L R$ & $R$ & $R$ \\
$p_{2}$ & $R$ & $D R$ & $R$ & $D R$ & $L R$ & $D R$ & $L R$ & $R$ & $R$ \\
$p_{3}$ & $R$ & $D R$ & $L R$ & $D R$ & $L R$ & $D R$ & $L R$ & $R$ & $L R$ \\
$p_{4}$ & $D R$ & $R$ & $L R$ & $D R$ & $L R$ & $D R$ & $L R$ & $R$ & $L R$ \\
$p_{5}$ & $D R$ & $D R$ & $R$ & $D R$ & $L R$ & $D R$ & $L R$ & $R$ & $L R$ \\
$p_{6}$ & $D R$ & $D R$ & $L R$ & $D R$ & $L R$ & $D R$ & $L R$ & $R$ & $R$ \\
\hline
\end{tabular}

membership values relative for the descriptor being set to Red:

$$
\begin{array}{lll}
\gamma_{\bar{R}}\{0.00\}=\frac{0}{6}=0.00 & \gamma_{\bar{R}}\{0.08\}=\frac{0}{6}=0.00 & \gamma_{\bar{R}}\{0.17\}=\frac{1}{6}=0.17 \\
\gamma_{\bar{R}}\{0.25\}=\frac{2}{6}=\frac{1}{3}=0.33 & \gamma_{\bar{R}}\{0.33\}=\frac{6}{6}=1.00 & \gamma_{\bar{R}}\{0.50\}=\frac{3}{6}=\frac{1}{2}=0.50 \\
\gamma_{\bar{R}}\{0.67\}=\frac{2}{6}=\frac{1}{3}=0.33 & \gamma_{\bar{R}}\{0.83\}=\frac{0}{6}=0.00 & \gamma_{\bar{R}}\{1.00\}=\frac{0}{6}=0.00
\end{array}
$$

One can see that the membership value of 0.33 returns a degree of significance of $\gamma \bar{R}\{0.33\}=1$, indicating that it was the only value agreed upon by all, thus satisfying the Eq. (14). The membership value itself is related to $f_{8}$, for which the swatch had an RGB value of $[255,0,0]$, which by all accounts is absolutely red. The highest scoring membership contained within the upper approximation was 0.50 , which was related to $f_{9}$, for which the swatch had an RGB value of $[255,51,51]$, which is very close to being red. The collected consensus of the group is in keeping with the realistic expectations for such an example. The plot presented in Fig. 7 shows the collective visualisation of sets based on all the generated R-fuzzy sets for Example 2

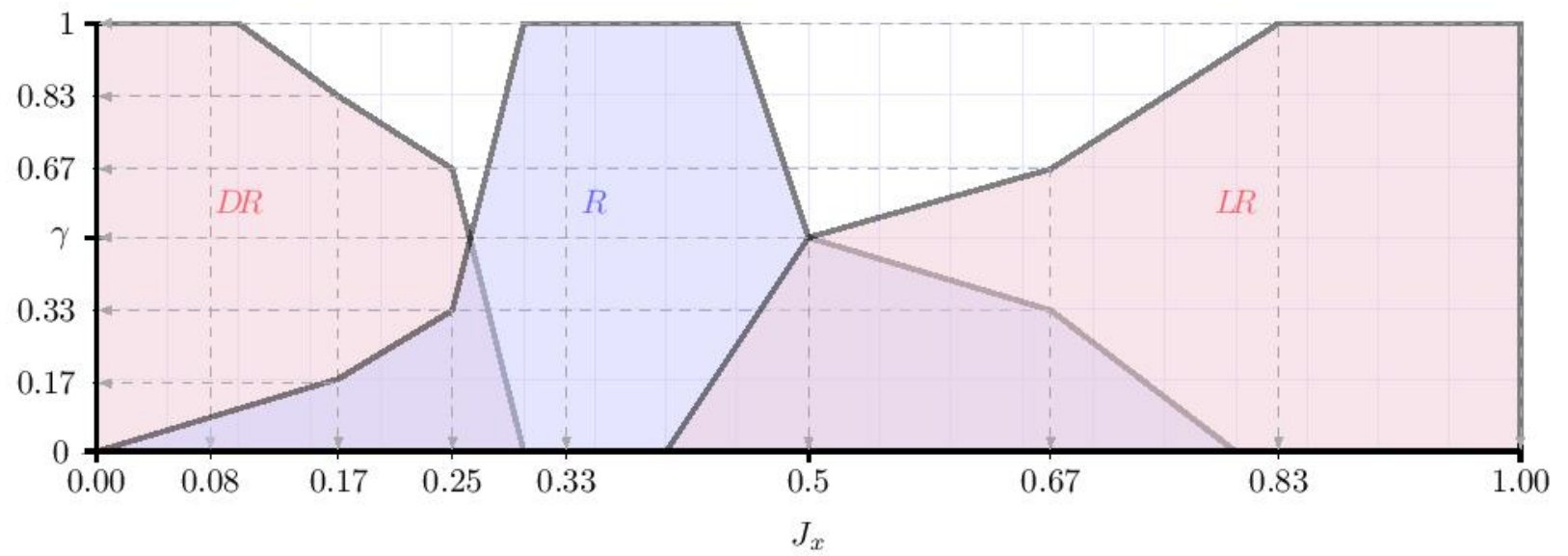

Figure 7: A continuous visualisation for Example 2, based on all the generated significance measures for the R-fuzzy sets of Light Red, Red \& Dark Red 


\subsection{Thresholds}

R-fuzzy sets could be used more intuitively if say, one wanted to know which membership values surpassed a particular threshold of acceptance. Assume $\Phi(x)>80$, where $\Phi$ is representative of a subset of membership values which evaluate to true based on the condition of exceeding a significance degree measure of 80 . As this would include all absolute lower approximation values (if such existed), it would also include membership values that were incredibly close to being included within the lower approximation. The significance degree measure for the membership value 0.57 for Example 1, when the descriptor was set to $d\left(f_{11}\right)=$ 'Acceptable', scored $\gamma \overline{A C}\{0.57\}=0.83$. This would exceed the threshold of $\Phi(x)>80$, and as a result would be treated as a lower approximation to some degree. Providing a threshold would alleviate the problem of allowing an outlier to stop a particular membership value from agreeing to the general consensus. The threshold value itself could be derived via heuristics, or simply a human expert or architect.

The extension of Example 1 via the significance measure, provides for highly detailed content. As an R-fuzzy set recognises and more importantly conserves the diversity inherent in human perception, the returned degrees of significance provide valuable information for airports and for people living within proximity. Conventional models would not be able to, or find it very difficult to conserve such diversity. Ignoring the importance of any relative membership value may lead to false noise exposure annoyance, which causes even more problems for residents. An individual value is not lost within an interval nor a shadowed area, it can be readily inspected and inferred from. Example 2, for much the same reason, allows for all perceptions; general and specific to be accounted for. Such an example could be further enhanced in the aiding of diagnosing visual impairments. Any human perception based environment becomes an applicable domain for an R-fuzzy and significance degree coupling. Even with a small sample, a consensus can still be achieved, providing a generalisation all the while still conserving each and every specific perception. In reality an R-fuzzy approach can be applied to a minimum of 2 people, from which the significance degree can create telling sets to encapsulate their perceptions. Any number of additional people and their perceptions can be integrated, forever evolving the significance degree measures and ergo, the shape the membership functions themselves.

\section{Relationships with fuzzy}

R-fuzzy sets are different from traditional fuzzy sets, in that their membership values are expressed as a set rather than a value. However, there are overlaps with others fuzzy variations under special considerations. In the original R-fuzzy paper by Yang and Hinde (2010), the relationships between R-fuzzy and type-1 fuzzy sets, interval-valued fuzzy sets and Atanassov intuitionistic fuzzy sets were given, with accompanying theorems and proofs, as such they have been omitted from this paper. This paper will put forward the relationship between that of the significance measure defined in Definition 6, and that of a type-1 fuzzy set. 
Also described is the relationship between an R-fuzzy set incorporated with a significance measure and that of a type-2 fuzzy set. It was remarked by Yang and Hinde, that if the distribution of membership functions could be modelled, they may then be used to derive a fuzzy set, which would give rise to an equivalent type-2 fuzzy set. As it has been shown in Section 3, the significance degree measure given in Eq. (13) does indeed act as an equivalent fuzzy set, when describing its descriptor. Albeit, with regards to its conditional probability of distribution.

Theorem 1. The significance measure described in Definition 6 is equivalent to a standard type-1 fuzzy set, if it can be described in the same way as presented in Definition 1. Whereby its membership function must satisfy the restriction imposed upon it, such that an object is assigned a degree of inclusion either equaling or within the range of $[0,1]$. Also for equivalence to be satisfied, the continuous set representation must be based upon the apex stick heights of the returned degrees of significance for the triggered membership values satisfying the descriptor being inspected.

Proof 1. From Definition 6 and Definition 5, assume that set $A$ is a descriptor for a particular R-fuzzy set. A traditional type-1 fuzzy set is a collection of ordered pairs. The degree of significance for each membership value belonging to a particular R-fuzzy set is quantified by its membership function $\gamma_{\bar{A}}\{v\}: J_{x} \rightarrow[0,1]$, such that it can be given by the expression:

$$
\bar{A}=\left\{\left\langle v, \gamma_{\bar{A}}\{v\}\right\rangle \mid v \in J_{x}\right\}
$$

Therefore, based on its descriptor the set will contain ordered pairs of membership values and their associated degrees of significance. One can see this expressions is equivalent to the notation given in Eq. (1):

$$
A=\left\{\left\langle x, \mu_{A}(x)\right\rangle \mid x \in \mathbb{U}\right\}
$$

(1 revisited)

Where an object is provided with a degree of inclusion relative to the set being inspected. Here we have $v \in J_{x}$ which is the membership set of membership values instead of $x \in \mathbb{U}$. As $J_{x}$ provides what essentially is the universe of discourse, the significance degree measure does indeed act as an equivalent type-1 fuzzy set, when the set is representative of the descriptor the R-fuzzy set was created for.

A type-2 fuzzy set is a logical extension to that of type-1, whereby the addition of a secondary grade of membership is used. The secondary grade itself is a type-1 fuzzy membership, and provides a threedimensional perspective, allowing for greater encapsulation of uncertainty.

Definition 7 (Type-2 fuzzy set (Mendel and John, 2002)): A type-2 fuzzy set $\tilde{A}$ is characterised by a type-2 membership function $\mu_{\tilde{A}}(x, u)$, where $x \in \mathbb{U}$ and $u \in J_{x} \subseteq[0,1]$. A type-2 fuzzy set is given by the formal expression:

$$
\tilde{A}=\left\{\left\langle(x, u), \mu_{\tilde{A}}(x, u)\right\rangle \mid \forall x \in \mathbb{U}, \forall u \in J_{x} \subseteq[0,1]\right\}
$$


in which $\mu_{\tilde{A}}: \mathbb{U} \times J_{x} \rightarrow[0,1] . \tilde{A}$ can also be expressed as:

$$
\tilde{A}=\int_{\forall x \in \mathbb{U}} \int_{\forall u \in J_{x} \subseteq[0,1]} \mu_{\tilde{A}}(x, u) /(x, u)
$$
require a type- 2 membership in a type-2 fuzzy set representation. Type-1 membership can be linked with objective measurements, but type-2 membership is much more subjective and lacks a reliable methodology 
to set it up. Therefore, this paper presents a practical and an effective methodology to conduct the work requiring a type-2 representation. From this perspective, an R-fuzzy and significance measure approach allows for an equivalent, almost intermediary approach, to ascertain higher details of resolution that a type2 fuzzy approach could capture, without the burden of high computational overhead and complexity of representation.

As Example 1 \& Example 2 have demonstrated, perception does not necessarily follow a strict and stringent train of thought, an individual may hold a perception that goes against the grain of the general norm. An informative representation of a descriptive term should satisfy not only the requirements of the imprecise representation, but also convey both the common perceptions and individual perceptions; the very ethos that R-fuzzy was created upon.

The main weakness for the proposed research is not in its configuration, but rather with regards to the criteria set $C$ and the predefined domain of the fuzzy membership set $J_{x}$. If either $C$ or $J_{x}$ subsequently change, the result of the R-fuzzy set would also change, this could be problematic for certain applications. Ideally, one would prefer to make use of the same criteria set of observers for a particular event, from which the predefined domain of the fuzzy membership set $J_{x}$ is established.

The problem of having to use excessive precision to describe increasingly imprecise phenomena has seen several approaches created to try to resolve this dilemma. To some extent these new approaches such as; interval-valued fuzzy sets, Atanassov intuitionistic fuzzy sets, shadowed sets and type-2 fuzzy sets, do offer viable solutions but not wholeheartedly, as several questions still remain. The theoretical relationships between type-2 fuzzy sets and R-fuzzy sets are established in this paper, and the relationships between the other aforementioned models and R-fuzzy sets are provided in the original work by Yang and Hinde (2010). The likes of interval-valued fuzzy sets, Atanassov intuitionistic fuzzy sets and shadowed sets, all allow for the means of encapsulating the uncertainty involved concerning the membership values of a fuzzy set. However, an interval-valued fuzzy set implies that the values contained within its interval are equally distributed; this is an unrealistic assumption for perception-based domains. Our examples have shown that unified distribution is not always the case; values may have a different relationship with the membership values concerned, whereas others may involve uncertainty. Other pitfalls are that a value can lose its inherent meaning if placed in shadowed regions or intervals, once placed in such a container, its uniqueness is diminished.

From Example 1 \& Example 2, one can see that perception based perspectives may not follow a universal interpretation, individuals may give varying results based on the same observations. These differences and similarities in their perceptions should all be taken into account. With this being the case, a single fuzzy membership value cannot be used to represent a descriptive object, doing so would skew the underlying intent of the perceptions involved. The general consensus and the individual perceptions need to be taken into consideration. A type-2 fuzzy approach extends into the third dimension by using a type-1 fuzzy set to 
replace the use of crisp membership values. Nevertheless, the secondary membership function itself would still be using crisp values, as a result we are faced with the same initial problem. However, the higher levels of type- $n$ one could implement, the closer one gets to precision and an agreed upon model, but not without consequence; the burden of complexity and computation becomes too costly.

The type-2 fuzzy set as presented in Definition 7 is the general interpretation, it has since been simplified somewhat as to allow for all possible membership values belonging to the secondary grade to have a value of 1 , referred to as an interval type-2 fuzzy set. As no new information is contained in this third dimension, it is ignored and instead the footprint of uncertainty ${ }^{1}$ is used to describe it. An interval type-2 fuzzy set makes use of interval mathematics, therefore making it easier to understand and compute compared to that of the generalised version. The review contained in Melin and Castillo (2014) concisely inspected type-2 fuzzy logic applications in the areas mainly involving clustering, classification and pattern recognition, with the vast majority of them involving interval type-2 fuzzy logic.

There are indeed some interesting methods that exist for constructing interval-valued fuzzy sets. The work undertaken by Bustince Sola et al. (2015) shows that an interval-valued fuzzy set is a particular case of an interval type-2 fuzzy set. As such, both concepts should be treated differently from one another. The sentiment of the paper is echoed by Mendel et al. (2016), reinforcing the perspective that they should indeed be treated differently.

As the membership of an R-fuzzy set itself is a set which contains discrete data, there is no loss of detail, unlike that of an interval valued fuzzy set approach. Once you are in the interval, you have no sense of how close to the bound of that interval you may be; extremely pessimistic or overtly optimistic, the interval assumes generality and uniformed distribution. As the membership set of an R-fuzzy set is a rough set, the contents of which are crisply defined possible fuzzy membership values, that have an affinity to the descriptor it is being modelled for, no loss of information is suffered. Therefore, one can then quantify the distribution of that R-fuzzy set, ergo the proposed significance measure that this paper puts forward.

Once the significance measure has been applied to the contained fuzzy memberships values of an R-fuzzy set, one could then use what would be equivalent to an $\alpha$-plane to provide horizontal slices. Given that the number of observations for a particular R-fuzzy set remains the same, this ensures that the same increments of significance are adhered to, even if one was to create a new R-fuzzy set with a different descriptor for the same data set, as can be seen from the examples. Where the final figures, Fig. 6 \& Fig. 7, are a collection of all the generated R-fuzzy sets where the descriptor is incremented.

From the enhanced example 1, there are 6 individuals for the criteria set $C$, this allows for the following possible degrees of significance to be registered; $\{0,0.17,0.33,0.5,0.67,0.86,1\}(0 / 6,1 / 6,2 / 6, \ldots, 6 / 6)$. With regards to example 1 , the two extremes are 0 and 1 . To obtain a significance of 1 , all in the populace must

\footnotetext{
${ }^{1}$ It is advisable one refers to the work of Mendel and John (2002), for a more thorough understanding.
} 
agree. Therefore, we should take 0.86 , as the extreme for the highest grade before absolute inclusion. Also, we should consider 0.17 as the other extreme, because a value of 0 indicates absolute non-inclusion. 0.17 can then be seen as the lowest possible grade before exclusion from the R-fuzzy set. Given our possible instances of significance, we can create an $\alpha$-plane with a horizontal slice at each instance. In much the same way that each $\alpha$-plane signifies the strength of significance, one could be interested in the extremes, 0.17 and 0.86 . As the membership of an R-fuzzy set is a rough set with an accompanying lower and upper approximation, the membership values associated to the lowest extreme $\alpha$ could be resigned to non-inclusion. Whereas the membership values assigned to the highest extreme $\alpha$ could be elevated to the lower approximation, which is an absolute agreement and inclusion. Using this perspective, whereby we essentially have three criteria, non-inclusion (0), total acceptance (1) and upper approximation inclusion to a varying degree $[0,1]$, we have replicated a shadow set approach. Where possible membership values in the extremes are given either as a 1 or 0 , and the values in the shadow regions simply belong to the interval $[0,1]$. You could argue the point, why would you go to the effort of calculating the significance for each of the contained fuzzy membership values if you are not going to use them? Given certain based perception domains, this would be beneficial, knowing what membership values would be deemed acceptable may be all the information ones needs rather than the exact degree of its significance. In particular, one would be more concerned with the values that were on the precipice of totally being agreed upon by all. Simply using the idea of $\alpha$-planes having their horizontal slices at the varying possible degrees of significance, would provide one with a great deal of detail regarding the domain the R-fuzzy set was modelled for. This undoubtedly allows for better uncertainty management, an ethos of higher uncertainty based approaches.

The approach that this paper puts forwards allows for the distribution of the contained elements that constitute the membership of an R-fuzzy set. As it contains discrete data, each and every contained membership value can be inspected and quantified. Once the significance degrees have been calculated, one can then apply an arbitrary (at least in terms of defining the left and right footprint parameters) membership function to encapsulate all the generated fuzzy membership values for a particular R-fuzzy set, as can be seen in Fig. 2 Fig. 3 Fig. 4 \& Fig. 5. This allows for a continuous approach to be adopted. By that, the combined R-fuzzy sets, for example Fig. 6, which coherently models the entire collective perception, a value which was not necessarily contained in the membership set for any generated R-fuzzy set, will still be able to provide a value of significance. For instance, the R-fuzzy set that modelled Acceptable AC, given in Fig. 2 , contained the fuzzy membership value 0.43 in its lower approximation, hence why it has an absolute value of 1 for its significance. The value 0.57 was also contained within the lower approximation, as it also has a significance of 1 . The plateau region between these two particular membership values should also allow for a potential membership value to return a degree of significance of 1 . If one was to inspect Fig. 2 or Fig. 6 , even though the membership value 0.5 was not included nor was it ever generated as a potential fuzzy membership value, but assuming that it was, it too would also return a degree of significance of 1 . This is 
true, as it between 0.47 and 0.57 , and contained within the plateau region of the arbitrary shape given to the R-fuzzy set.

Using the approach described in this paper, of coupling together an R-fuzzy set and the proposed significance measure, one is able to garner higher levels of detailed information, all the while retaining the sentiment from individual and collective perceptions. The burden of complexity and computation is minimal compared to a full type-2 approach, and as such, we believe our approach to be far superior in modelling perceptions than any of the aforementioned concepts, particularly type-2 fuzzy.

\section{Conclusion}

This paper has set about describing the effectiveness of using an R-fuzzy approach, coupled with the newly derived significance measure for encapsulating fuzzy membership values, when precise memberships are not known. The various extensions of fuzzy set theory do not alleviate this problem in its entirety, instead the significance of the value is lost in the shadow areas or intervals themselves. However, a type-2 fuzzy approach is very close to an ideal concept, albeit, the associated complexity and burden of precision makes it unfavourable. An R-fuzzy set is a relatively new extension of fuzzy set theory, one that allows for the encapsulation and representation of membership values, which would be difficult to capture using more traditional means, allowing for the variance of relative perceptions to be captured and their uniqueness to be maintained. The proposed significance measure is based on that of the standard rough certainty factor described in Eq. (12), from which a relative significance measure can be derived, Eq. (13). One which is indicative of $J_{x}$ and the descriptor based on the rough membership set generated. Understanding the importance of the membership values contained within the upper approximation allows for a better understanding on the perception being modelled.

One of the distinctions between intelligent systems and other systems is its ability in dealing with uncertainty. Uncertainty in fuzzy memberships is a common problem in the application of fuzzy sets, which lead to the concept of type-2 fuzzy sets. Type-2 fuzzy sets have a strong theoretical capacity in uncertainty representation, but its difficulty is with regards to its highly subjective type-2 membership. Also, its computational complexity limits its applicability and application domain. By connecting R-fuzzy sets and type-2 fuzzy sets through the newly defined significance measure, this paper has proposed a new way to solve the challenge of type-2 fuzzy sets in its applications. This was done by replacing the subjective membership with a collection of objective type-1 membership values in an R-fuzzy set. In this way, the precision of type-2 fuzzy sets are preserved but its difficulty in defining type-2 membership is removed.

As mentioned in the earlier in the paper, the sensitivity of the result of R-fuzzy sets with regards to the criteria set $C$ and the domain $J_{x}$, may act as a restriction to its application in some real world problems domains, where it might be hard to construct a comprehensive $C$ and $J_{x}$ in the early stages of the inspection. 
On the other hand, there is no standard framework to construct $C$ and $J_{x}$ for R-fuzzy sets at the moment. Furthermore, in theory, type-2 fuzzy sets could go to type- $n$, but our model here applies only to type-2 fuzzy sets.

Therefore, a number of related future research directions can be foreseen from this research. A systematic investigation into the methodology to establish the criteria set $C$ and domain $J_{x}$ needs to be carried out to establish a practical framework under different application domains. The sensitivity of R-fuzzy sets on its associated $C$ and $J_{x}$ needs to be further studied to identify a suitable strategy to minimise its impact on the results. Although this paper established the relationship between R-fuzzy sets and type-2 fuzzy sets, the relationship between R-fuzzy sets and type- $n$ fuzzy sets can be further explored. In addition to the higher precision for uncertain memberships, the application of R-fuzzy sets to group decision making is also an interesting research topic for further investigation. The application domain of type-2 fuzzy, where either the general or interval interpretation is used, would also be an ideal research interest. Type-2 fuzzy has excellent capacity in dealing with uncertainty, but the difficulty in defining its fuzzy sets of membership limits its applicability. With the proved connections between R-fuzzy sets \& the significance measure, to that of type-2 fuzzy sets, a type-2 fuzzy problem can be converted to an R-fuzzy set problem. In which case, R-fuzzy sets can be applied to solve problems where type-2 fuzzy sets are currently used, or where it would be preferable to use a type-2 approach. Therefore a typical type- 2 application domain, once converted to an R-fuzzy set, becomes an application domain of an R-fuzzy approach.

\section{References}

Atanassov, K. T. (1986). Intuitionistic fuzzy sets. Fuzzy Sets and Systems, 20(1):87 - 96.

Balas, C. E., Ko, M. L., and Tr, R. (2010). Artificial neural networks based on principal component analysis, fuzzy systems and fuzzy neural networks for preliminary design of rubble mound breakwaters. Applied Ocean Research, $32(4): 425$ - 433.

Berenji, H. R. (1988). Treatment of uncertainty in artificial intelligence. In Machine intelligence and autonomy for aerospace systems, pages $233-247$.

Bodjanova, S. (2007). Granulation of a fuzzy set: Nonspecificity. Information Sciences, 177(20):4430 - 4444.

Bosque, G., del Campo, I., and Echanobe, J. (2014). Fuzzy systems, neural networks and neuro-fuzzy systems: A vision on their hardware implementation and platforms over two decades. Engineering Applications of Artificial Intelligence, 32(0):283 331.

Bustince Sola, H., Fernandez, J., Hagras, H., Herrera, F., Pagola, M., and Barrenechea, E. (2015). Interval type-2 fuzzy sets are generalization of interval-valued fuzzy sets: Toward a wider view on their relationship. Fuzzy Systems, IEEE Transactions on, 23(5):1876-1882.

Cantor, G. (1895). Beitrge zur begrndung der transfiniten mengenlehre. Mathematische Annalen, 46(4):481 - 512.

Coletti, G. and Scozzafava, R. (2004). Conditional probability, fuzzy sets, and possibility: a unifying view. Fuzzy Sets and Systems, 144(1):227 - 249. Possibilistic Logic and Related Issues.

Deng, T., Chen, Y., Xu, W., and Dai, Q. (2007). A novel approach to fuzzy rough sets based on a fuzzy covering. Information 
Deschrijver, G. and Kerre, E. E. (2003). On the relationship between some extensions of fuzzy set theory. Fuzzy Sets and Systems, 133(2):227 - 235 .

Drakopoulos, J. A. (1995). Probabilities, possibilities, and fuzzy sets. Fuzzy Sets and Systems, 75(1):1 - 15.

Dubois, D. and Prade, H. (1990). Rough fuzzy sets and fuzzy rough sets*. International Journal of General Systems, 17(23):191-209.

Dubois, D. J. (1980). Fuzzy sets and systems: theory and applications, volume 144. Academic press.

Huynh, V.-N. and Nakamori, Y. (2005). A roughness measure for fuzzy sets. Information Sciences, $173(13): 255$ - 275.

Jensen, R. and Shen, Q. (2008). Computational intelligence and feature selection: rough and fuzzy approaches, volume 8. John Wiley \& Sons.

Jensen, R. and Shen, Q. (2009). New approaches to fuzzy-rough feature selection. Fuzzy Systems, IEEE Transactions on, $17(4): 824-838$.

Klir, G. and Folger, T. (1988). Fuzzy Sets, Uncertainty, and Information. Physica-Verlag.

Klir, G. and Wierman, M. (1998). Uncertainty-Based Information. Physica-Verlag.

Lesniewski, S. (1929). Grundzge eines neuen systems der grundlagen der mathematik. Fundamenta Mathematicae, 14(1):1-81.

Li, Q., Wang, P., and Lee, E. (1996). r-fuzzy sets. Computers \& Mathematics with Applications, $31(2): 49$ - 61.

Lian, R.-J. (2012). Grey-prediction self-organizing fuzzy controller for robotic motion control. Information Sciences, 202(0):73 $-89$.

Lin, F.-J. and Wai, R.-J. (2002). Hybrid computed torque controlled motortoggle servomechanism using fuzzy neural network uncertainty observer. Neurocomputing, 48(14):403-422.

Melin, P. and Castillo, O. (2014). A review on type-2 fuzzy logic applications in clustering, classification and pattern recognition. Applied Soft Computing, 21(0):568 - 577.

Melin, P., Gonzalez, C., Castro, J., Mendoza, O., and Castillo, O. (2014). Edge-detection method for image processing based on generalized type-2 fuzzy logic. Fuzzy Systems, IEEE Transactions on, 22(6):1515-1525.

Mendel, J., Hagras, H., Bustince, H., and Herrera, F. (2016). Comments on "interval type-2 fuzzy sets are generalization of interval-valued fuzzy sets: Towards a wide view on their relationship";. Fuzzy Systems, IEEE Transactions on, 24(1):249250 .

Mendel, J. and John, R. (2002). Type-2 fuzzy sets made simple. Fuzzy Systems, IEEE Transactions on, 10(2):117-127.

Mendel, J., Liu, F., and Zhai, D. (2009). $\alpha$-plane representation for type-2 fuzzy sets: Theory and applications. Fuzzy Systems, IEEE Transactions on, 17(5):1189-1207.

Mendes, J., Souza, F., Araújo, R., and Gonçalves, N. (2012). Genetic fuzzy system for data-driven soft sensors design. Applied Soft Computing, 12(10):3237 - 3245

Nanda, S. and Majumdar, S. (1992). Fuzzy rough sets. Fuzzy Sets and Systems, 45(2):157 - 160.

Nasab, H. H. (2014). A hybrid fuzzy-ga algorithm for the integrated machine allocation problem with fuzzy demands. Applied Soft Computing, 23(0):417 - 431 .

Nasir, M., Lim, C. P., Nahavandi, S., and Creighton, D. (2014). A genetic fuzzy system to model pedestrian walking path in a built environment. Simulation Modelling Practice and Theory, 45(0):18 - 34 .

Nguyen, H. T. (1997). Fuzzy sets and probability. Fuzzy Sets and Systems, 90(2):129 - 132. Fuzzy Sets: Where Do We Stand? Where Do We Go?

Otadi, M. (2014). Fully fuzzy polynomial regression with fuzzy neural networks. Neurocomputing, 142(0):486 - 493. \{SI\} Computational Intelligence Techniques for New Product Development.

Pawlak, Z. (1982). Rough sets. International Journal of Computer ES Information Sciences, 11(5):341-356.

Pawlak, Z. (1998). Rough set theory and its applications to data analysis. Cybernetics \& Systems, 29(7):661-688.

Pawlak, Z. (2002). Rough sets and intelligent data analysis. Information Sciences, 147(14):1 - 12. 
Pawlak, Z. and Skowron, A. (2007). Rough sets: Some extensions. Information Sciences, 177(1):28 - 40. Zdzis?aw Pawlak life and work (19262006).

Pedrycz, W. (1998). Shadowed sets: representing and processing fuzzy sets. Systems, Man, and Cybernetics, Part B: Cybernetics, IEEE Transactions on, 28(1):103-109.

Pedrycz, W., Han, L., Peters, J., Ramanna, S., and Zhai, R. (2001). Calibration of software quality: Fuzzy neural and rough neural computing approaches. Neurocomputing, 36(14):149 - 170. Rough-neuro computing.

Pourahmadi-Nakhli, M., Seifi, A. R., and Taghavi, R. (2013). A nonlinear-hybrid fuzzy/probabilistic load flow for radial distribution systems. International Journal of Electrical Power \& Energy Systems, 47(0):69 - 77.

Purba, J. H. (2014). Fuzzy probability on reliability study of nuclear power plant probabilistic safety assessment: A review. Progress in Nuclear Energy, 76(0):73 - 80.

Radzikowska, A. M. and Kerre, E. E. (2002). A comparative study of fuzzy rough sets. Fuzzy Sets and Systems, 126(2):137 155.

Rajmohan, T., Palanikumar, K., and Prakash, S. (2013). Grey-fuzzy algorithm to optimise machining parameters in drilling of hybrid metal matrix composites. Composites Part B: Engineering, 50(0):297 - 308.

Roisenberg, M., Schoeninger, C., and da Silva, R. R. (2009). A hybrid fuzzy-probabilistic system for risk analysis in petroleum exploration prospects. Expert Systems with Applications, 36(3, Part 2):6282 - 6294 .

Salmeron, J. L. and Gutierrez, E. (2012). Fuzzy grey cognitive maps in reliability engineering. Applied Soft Computing, 12(12):3818 - 3824. Theoretical issues and advanced applications on Fuzzy Cognitive Maps.

Sambuc, R. (1975). Fonctions $\Phi$ foues. Application a l'aide au Diagnostic en Patholologie Thyroidienne. PhD thesis, Univ. Marseille, France.

Sun, B., Ma, W., and Chen, D. (2014). Rough approximation of a fuzzy concept on a hybrid attribute information system and its uncertainty measure. Information Sciences, 284(0):60 - 80. Special issue on Cloud-assisted Wireless Body Area Networks.

Wagner, C. and Hagras, H. (2010). Toward general type-2 fuzzy logic systems based on zslices. Fuzzy Systems, IEEE Transactions on, 18(4):637-660.

Wagner, C. and Hagras, H. (2013). Advances in Type-2 Fuzzy Sets and Systems: Theory and Applications, chapter zSlices Based General Type-2 Fuzzy Sets and Systems, pages 65-80. Springer New York, New York, NY.

Wu, W.-Z., Mi, J.-S., and Zhang, W.-X. (2003). Generalized fuzzy rough sets. Information Sciences, $151(0): 263$ - 282.

$\mathrm{Xu}$, W., Liu, Y., and Sun, W. (2012). Intuitionistic fuzzy rough sets model based on ( $\theta$, $\phi)$-operators. In Fuzzy Systems and Knowledge Discovery (FSKD), 2012 9th International Conference on, pages 234-238. IEEE.

Yang, Y. and Hinde, C. (2010). A new extension of fuzzy sets using rough sets: R-fuzzy sets. Information Sciences, 180(3):354 -365 .

Yao, Y. (1996). Two views of the theory of rough sets in finite universes. International Journal of Approximate Reasoning, 15(4):291 - 317. Rough Sets.

Zadeh, L. (1975). The concept of a linguistic variable and its application to approximate reasoning-i. Information Sciences, $8(3): 199-249$.

Zadeh, L. (1999). Fuzzy sets as a basis for a theory of possibility. Fuzzy Sets and Systems, 100, Supplement 1(0):9 - 34.

Zadeh, L. A. (1965). Fuzzy sets. Information and control, 8(3):338-353.

Zadeh, L. A. (1972). A fuzzy-set-theoretic interpretation of linguistic hedges. Cybernetics, 2(3):4-34.

Zeng, A., Li, T., Liu, D., Zhang, J., and Chen, H. (2015). A fuzzy rough set approach for incremental feature selection on hybrid information systems. Fuzzy Sets and Systems, 258(0):39 - 60. Special issue: Uncertainty in Learning from Big Data. 